\title{
Breakup of Air Bubbles in Water: Memory and Breakdown of Cylindrical Symmetry
}

\author{
Nathan C. Keim, * Peder Møller, Wendy W. Zhang, and Sidney R. Nagel \\ James Franck Institute, University of Chicago, 929 East 57th Street, Chicago, Illinois 60637, USA
}

(Received 26 May 2006; published 3 October 2006)

\begin{abstract}
Using high-speed video, we have studied air bubbles detaching from an underwater nozzle. As a bubble distorts, it forms a thin neck which develops a singular shape as it pinches off. As in other singularities, the minimum neck radius scales with the time until the breakup. However, because the air-water interfacial tension does not drive the breakup, even small initial cylindrical asymmetries are preserved throughout the collapse. This novel, nonuniversal singularity retains a memory of the nozzle shape, size, and tilt angle. In the last stages, the air appears to tear instead of pinch.
\end{abstract}

DOI: 10.1103/PhysRevLett.97.144503

PACS numbers: 47.55.db, 02.40.Xx, 47.55.df

The delightful tingling felt when drinking carbonated beverages, the glee of children blowing bubbles in a bathtub, and the importance of deep underwater fissures venting gases into the oceans hint at the richness and significance of bubble formation in determining the texture and composition of our world. However, the process by which a bubble is formed is still full of surprises. A drop or bubble breaks up by forming a neck that thins to atomic dimensions, a process described as an approach towards a singularity where physical quantities such as stress or pressure grow infinitely large. Singularities often organize the overall dynamical evolution of nonlinear systems. Each symmetry in nature implies an underlying conservation law, so that the symmetries of the singularity associated with pinch-off naturally have important consequences for its dynamics. It was previously believed [1-12] that the pinching neck of any drop or bubble would become cylindrically (i.e., azimuthally) symmetric in the course of pinch-off. Recently, pinching necks of air in water were observed to lose cylindrical symmetry in the course of detachment $[13,14]$.

Here we show that this loss of symmetry is caused by a new form of memory in singular dynamics: Even a small asymmetry in the initial conditions is preserved throughout bubble detachment. This novel singularity retains a memory of the nozzle shape, size, and tilt angle. The asymmetry can be made so great that the air appears to tear. This symmetry breaking may be important in numerous applications [15-17] and for understanding other physical processes which are modeled as the formation of a singularity, such as star or black hole formation [18] and supernova explosions [19]. Thus, our experimental observation of the breakdown of cylindrical symmetry in the air bubble demonstrates a new view of dynamical singularities that may be relevant even on a celestial scale.

Singularities govern the dynamics in many familiar breakup events, such as the dispersal of oil drops into vinegar during the making of a salad dressing or the dripping of water from a leaky faucet. For many fluid pairs for example, one viscous fluid breaking in a surrounding fluid of high viscosity $[7,8,12]$ — the shape and dynamics of the pinching neck depend solely on the fluid parameters, as the breakup forgets its initial conditions on approaching the singularity. Such universal behavior, where the dynamics of pinch-off are dominated solely by the singularity that lies at its end, was until recently thought to be the only way in which a fluid could break apart. However, Doshi et al. [20] discovered an exceptional form of pinch-off when an inviscid fluid pinches off inside a viscous one. Here the axial curvature of the neck is preserved and a change in nozzle size is remembered throughout the breakup. That any memory of initial conditions persists is surprising and raises new questions about the possible types of dynamics near a singularity: During pinch-off, how much and what kind of information can be remembered about initial conditions, and how does this memory influence the permanent structures that appear after the singularity has been formed?

In this Letter, we show that, when an air bubble breaks off from a submerged nozzle, not only does the pinching neck of air retain an indelible imprint of its initial geometry, but the initial azimuthal asymmetry alters the number and subsequent trajectories of satellite bubbles. Extreme asymmetries in the initial shape of the air bubble result in a new, fully three-dimensional mode of pinch-off, in which the air tears apart in successive sharp jerks, instead of pinching at a point.

Normally, surface tension can be relied upon to restore the cylindrical symmetry of a pinching liquid neck even when the initial conditions are asymmetric. However, as first pointed out by Longuet-Higgins et al. [21] and subsequently elaborated upon by experiments and simulations $[13,14,22-26]$, the detachment of an air bubble from a nozzle is not a collapse driven by surface tension but rather is an implosion due to a pressure difference between the hydrostatic pressure in the water and the bubble pressure, $\Delta P \psi=\Delta \rho g a$. Here $a \psi$ s the linear size of the bubble:

$$
a \psi=\left(\frac{R_{N} \sigma}{g \Delta \rho}\right)^{1 / 3}, \psi
$$


where $R_{N \psi}$ is the nozzle radius, $\sigma \psi$ s the surface tension, $\Delta \rho \psi$ is the difference in the densities of the liquid and the air, and $g$ \& s the gravitational acceleration [23]. The relevant experiment, to indicate if surface tension plays a role in the asymptotic dynamics, is to measure the radius of the neck of air at its narrowest part $h_{\min }$ as a function of $\tau$, the time left to the singularity. If the implosion dynamics persist until the breakup, then

$$
h_{\min }=\phi\left(a^{3} g\right)^{1 / 4} \tau^{\alpha_{h}}, \psi
$$

where $\beta \psi$ s a numeric prefactor and $\alpha_{h \psi}=4 / 2$.

Burton et al. [23] reported excellent experimental agreement with this result. We note here that surface tension sets the initial size of the bubble but plays no role in the dynamics. This, therefore, sets bubble detachment apart from all other breakup situations studied so far, where one or both fluids are viscous or where two inviscid fluids differ little in density, as well as from the inverse case of water in air. In those cases, the breakup is driven by surface tension and $\alpha_{h \psi} \geq z / 3[3-12,20]$. Here we observe the consequences of a different driving mechanism on the breakup dynamics.

To create bubbles, we use a syringe pump to release air from circular underwater nozzles with radii $R_{N}$ from 1.5 to $4.1 \mathrm{~mm}$. The nozzle and water tank rest on a precision twoaxis tilting platform, which allows us to break and restore cylindrical symmetry. In contrast to Bergmann et al. [14], our bubbles from circular nozzles are produced quasistatically $\left(0.03 \mathrm{~s}^{-1}\right)$. The growing bubble becomes unstable and begins to detach when the Bond number is approximately 1 . Oblong nozzles are also used to introduce more extreme asymmetries. Bubbles are backlit and photographed with a Vision Research "Phantom" version 7 camera at rates up to 130000 frames/s. For each video frame, a computer traces the neck profile and obtains $h_{\min }$.

Burton et al. [23] reported the remarkable observation that, instead of proceeding smoothly to zero radius, the neck of air abruptly breaks apart in what they term a "rupture" at $h_{\min } \approx 25 \mu \mathrm{m}$, which they attribute to a Kelvin-Helmholtz instability that is intrinsic to the dynam-

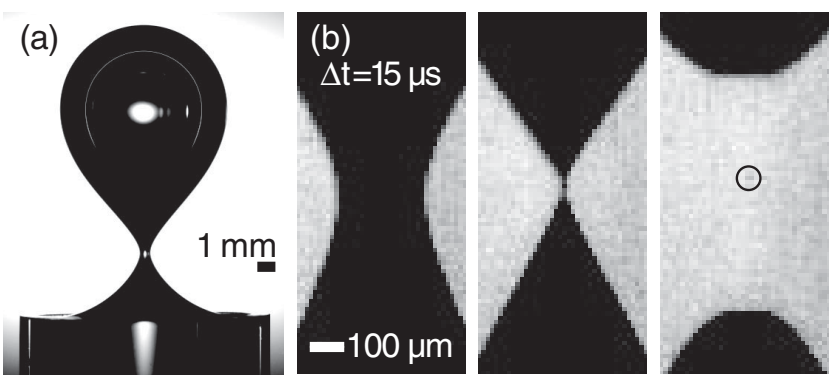

FIG. 1. Pinch-off of an air bubble from a level underwater, circular nozzle with radius $R_{N \psi}=4.1 \mathrm{~mm}$. (a) The bubble appears dark, except for bright optical artifacts. (b) A magnified sequence of pinch-off. In the last frame, the single $\sim 5 \mu \mathrm{m}$ satellite bubble is circled. $\Delta$ tygives the time between frames. ics. However, as shown in Fig. 1, when the nozzle is carefully leveled, we find that the pinch-off appears to be cylindrically symmetric and proceeds, without rupture, to scales below our camera resolution $(\sim 4 \mu \mathrm{m})$. Figure 2 shows $h_{\min }$ versus $\tau \psi$ for two nozzles. The data are well fit with a power law: $h_{\min } \alpha \tau^{\alpha_{h}}$, with $\alpha_{h \psi}=\oplus .56 \pm 0.03$. This is consistent with the simulations of Leppinen et al., who found $\alpha_{h \psi} \approx \theta .55$ [25] but exclude $\alpha_{h \psi}=\oplus .50$ [21-23]. Our data cannot distinguish pure power-law behavior with $\alpha_{h \psi}=4.56$ (solid lines in Fig. 2) from a power law $\alpha_{h \psi}=\longleftarrow$ 0.50 with logarithmic corrections (dotted lines) that have been derived for Eq. (2) [14,24] - a limitation also encountered by Bergmann et al. at low Froude numbers. However, these corrections are derived by assuming a slender cylinder, which we believe is a poor approximation to our neck shape. The pure power-law prefactor scales approximately as $R_{N \psi}^{0.25}$, as predicted by Eqs. (1) and (2). In a forthcoming paper, we will present a more detailed analysis of the dependence on $R_{N \psi}$ and $\sigma \psi$ and the scaling of the entire neck profile in both the radial and axial directions [27]. Here the exponent $\alpha_{h, \phi}$ s considerably smaller than $2 / 3$. We suggest that the neck of an air bubble could collapse so rapidly that the force due to surface tension would not keep pace with the evolving dynamics; hydrostatic pressure and Bernoulli pressure instead drive the breakup [21,28]. We now show that, as the breakup is approached, surface tension is indeed no longer effective at erasing cylindrical asymmetry and that this has profound effects.

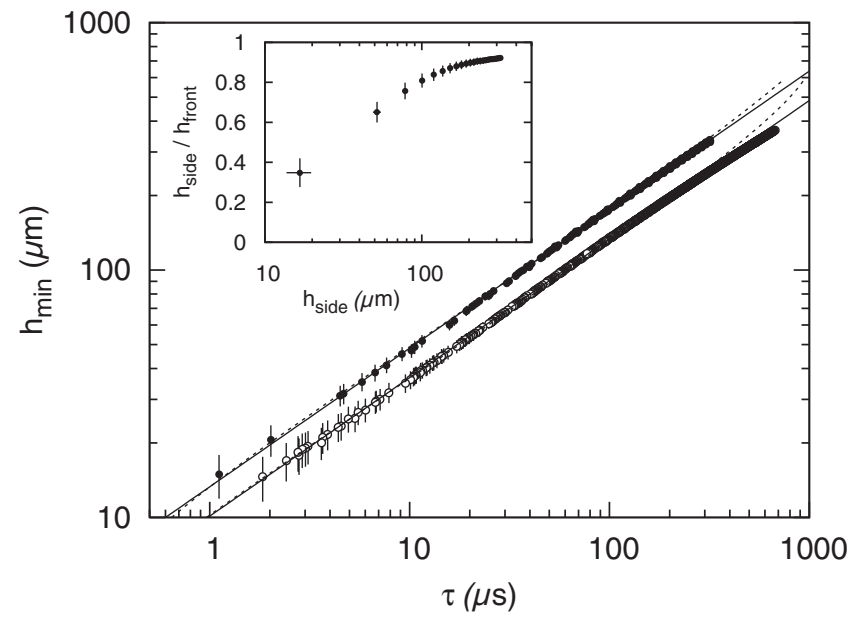

FIG. 2. Scaling of $h_{\min }$ versus $\tau$ for bubbles released quasistatically in water from circular nozzles of $R_{N \psi}=4.5 \mathrm{~mm}$ (open circles) and $4.1 \mathrm{~mm}$ (solid circles). The Reynolds numbers in water at the beginning of detachment are approximately 0.6 and 4 , respectively. The solid lines show power-law fits with exponent $\alpha_{h \psi}=\oplus .56$, and the dashed lines show fits with logarithmic corrections [14,24]. Data are taken from multiple pinch-offs, and only data for $\tau \psi<230 \mu \mathrm{s}$ are used for fitting. Inset: For the tilted-nozzle image sequences shown in Fig. 3(a), the radial size of the neck scales differently in the front $\left(h_{\text {front }}\right)$ and side $\left(h_{\text {side }}\right)$ directions. 

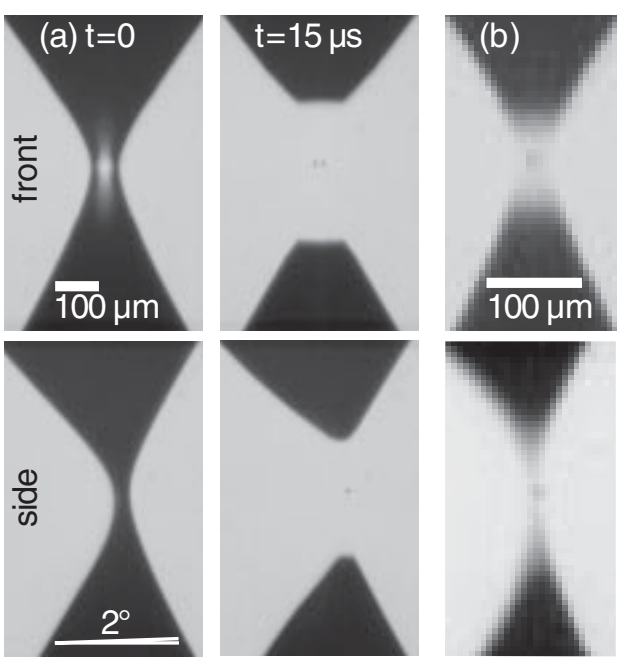

FIG. 3. Front and side views of pinch-off from an $R_{N \psi}=\longleftarrow$ $4.1 \mathrm{~mm}$ nozzle tilted by $2^{\circ}$. The nozzle's tilt is indicated in the bottom-left frame; in the front images, it is tilted away from the camera. Front and side images are from separate sequences but were selected to match length scales closely. (a) The neck is broadened before the breakup, resembling a crimped and bent double cone. A pair of satellite bubbles $15 \mu \mathrm{m}$ in diameter is produced. Afterward, the tip of each interface is bifurcated. (b) In these $2 \mu \mathrm{s}$ exposures, the final form of the neck is captured along with the initial positions of the satellite bubbles. The neck reaches zero thickness while it is still $20 \mu \mathrm{m}$ wide, resulting in what appears to be a rupture instead of a smooth, symmetric pinch-off.

We can test for a memory of asymmetry by tilting the nozzle slightly away from the vertical axis. Figure 3 represents pinch-off for tilt angles $\gtrsim 1^{\circ}$. Even this small tilt flattens the neck sufficiently to produce two satellite bubbles instead of the single one found with the leveled nozzle [as shown in the last panel in Fig. 1(b)]; the interface has an increasingly bifurcated appearance after pinch-off; and the breakup event is no longer centered above the nozzle but is shifted laterally away from the direction of tilt (i.e., rightward in the "side" views in Fig. 3). For these pinch-offs, $h_{\min }$ also scales as a power law, with $\alpha_{h \psi}=\theta .59 \pm 0.02$. Even deviations as small as $0.07^{\circ}$ from the vertical axis give rise to visible lateral asymmetry in the interface profile $100 \mu \mathrm{s}$ after pinch-off. This smaller tilt corresponds to a displacement of only $10 \mu \mathrm{m}$ at the rim of the $R_{N \psi}=4.1 \mathrm{~mm}$ nozzle used in Fig. 3. The effects of tilting are most pronounced for the larger nozzle. The rupture observed by Burton et al. [23] resembles our photographs taken with a small tilt (Fig. 3). We have seen that by carefully leveling the nozzle we can delay rupture to scales below our resolution $(4 \mu \mathrm{m})$; rupture is therefore unlikely to be caused by an intrinsic Kelvin-Helmholtz instability at $25 \mu \mathrm{m}$ as Burton et al. suggested.

Tilting the nozzle also changes scaling, as shown in the inset in Fig. 2 . $h_{\min }$ is replaced by $h_{\text {front }}$ and $h_{\text {side }}$, corresponding to the views in Fig. 3 . As $h_{\text {side }}$ becomes small, the ratio $h_{\text {side }} / h_{\text {front }}$ goes to zero. Above $10 \mu \mathrm{s}$ before the breakup, scaling is the same as for a level nozzle, with $\$ 10 \%$ difference in the power-law prefactor.

The satellite bubbles produced at the breakup serve as tracers to indicate subsequent liquid flow, as the buoyant rising of a $15 \mu \mathrm{m}$ bubble $\left(10^{-4} \mathrm{~cm} / \mathrm{s}\right)$ is negligible compared to the observed velocities $(80 \mathrm{~cm} / \mathrm{s})$. Following pinch-off from a nozzle tilted by $2^{\circ}$, satellite bubbles move upward and away from the direction of tilt (i.e., rightward in Fig. 3). This motion suggests a cylindrical asymmetry in fluid velocities around the breakup point: Water that has traveled farther to the breakup point is also moving faster. The pair of satellite bubbles produced from a nozzle tilted

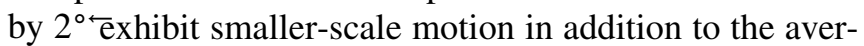
age motion just described: Bubbles may circle about each other laterally with a period $150 \mu \mathrm{s}$, indicating vertical vorticity.

That pinch-off is sensitive to small deviations from cylindrical symmetry suggests that gross asymmetry in initial conditions would lead to correspondingly dramatic outcomes. In addition, it could provide a visible example of a fully three-dimensional breakup. To test this, we used a nozzle with an oblong opening, a $9.6 \mathrm{~mm} \times 1.6 \mathrm{~mm}$ slot with rounded ends. If the time scale of bubble inflation is shorter than that for capillary waves ( $100 \mathrm{~ms}$ ), pinch-off begins asymmetrically. To achieve rapid inflation $(40 \mathrm{~ms})$,

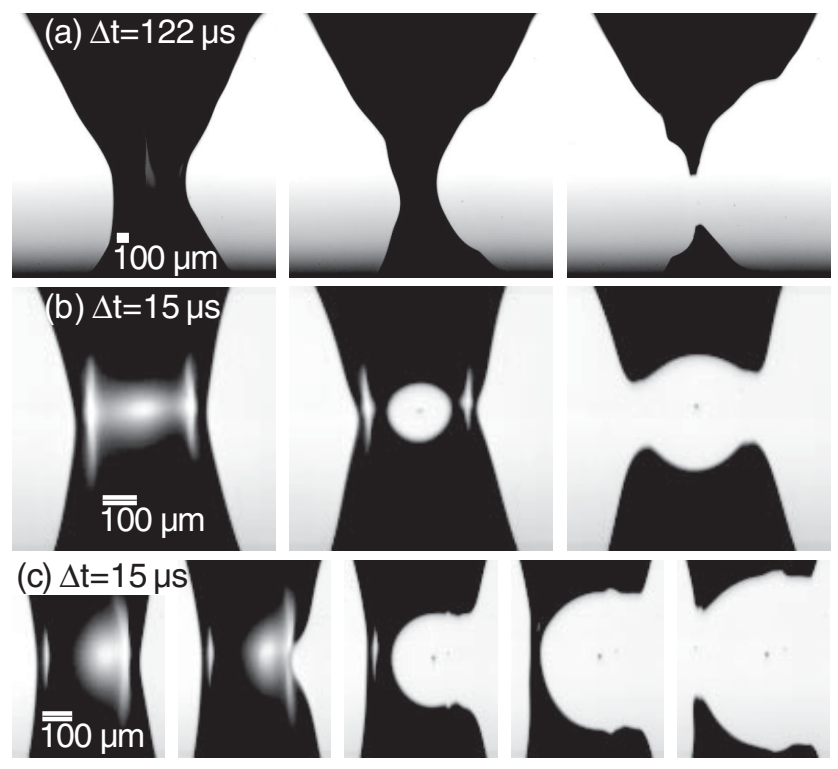

FIG. 4. Pinch-off of a burst of air from a slot-shaped nozzle, displaying strong cylindrical asymmetry. (a) For slower bursts, the neck is broadened with a scalloped profile. In a series of discrete events, it tears iteratively from one or both sides. (b) For faster bursts, the neck is more ribbonlike. The ribbon thins until coalescence is initiated, creating a hole in the center of the neck. The remaining two columns of air quickly break. Each of the topological transitions creates one or more satellite bubbles. (c) Under the conditions for (b), we also see off-center tearing that combines aspects of (a) and (b). 
we operate a valve to make small bursts of air from the syringe. The resulting necks have no semblance of cylindrical symmetry but are broadened in the same direction as the nozzle opening. Figure 4 shows three representative outcomes. At lower burst pressures, the neck is flattened and "tears" from its edges, resulting in a scalloped appearance that reflects a history of discrete events. At higher pressures, the neck becomes ribbonlike near pinch-off, thinning sufficiently to initiate coalescence near its center.

The pinch-off of air in water shows a radically new behavior: Any cylindrical asymmetry is preserved throughout the breakup process. Others have examined the effect of initial conditions on the evolution of the neck in a coflowing air-water jet [24] and for very large bubbles and found that the scaling exponent $\alpha_{h \psi}$ is nonuniversal [14]. Other singularities [1] have been shown to be sensitive to noise [2]. However, experiment and theory up to now have ignored cylindrical asymmetry [21-26] or could not show it to be a generic feature of breakup [14]. We have shown that tilting the nozzle by just $0.07^{\circ}$ detectably alters the outcome of the breakup. When the initial asymmetry is strongly exaggerated, the neck tears in two.

Because of its sensitivity to cylindrical asymmetry, the pinch-off of air in water demonstrates a kind of memory previously unanticipated in fluid pinch-off. Without surface tension, the detachment dynamics no longer converge to a cylindrically symmetric solution. When other physical processes are modeled as the formation of a singularity, we often assume that the singularity formation possesses all the symmetries allowed by the fundamental laws describing the physical process. However, it is not known whether such symmetries can be realized in the presence of arbitrary initial conditions. In the case of a detaching air bubble, we see that the dynamics do not assume the full symmetry allowed and that the generic breakup is threedimensional.

We thank Francois Blanchette, Justin Burton, Detlef Lohse, Peter Taborek, and Lei Xu for comments. This work was supported by NSF No. DMR-0352777 and MRSEC No. DMR-0213745.

*Electronic address: nkeim@uchicago.edu

[1] J. Eggers and T.F. Dupont, J. Fluid Mech. 262, 205 (1994).

[2] X. D. Shi, M.P. Brenner, and S. R. Nagel, Science 265, 219 (1994).
[3] J. Eggers, Rev. Mod. Phys. 69, 865 (1997).

[4] Y. J. Chen and P. H. Steen, J. Fluid Mech. 341, 245 (1997).

[5] R. F. Day, E. J. Hinch, and J. R. Lister, Phys. Rev. Lett. 80, 704 (1998).

[6] J. R. Lister and H. A. Stone, Phys. Fluids 10, 2758 (1998).

[7] W. W. Zhang and J. R. Lister, Phys. Rev. Lett. 83, 1151 (1999).

[8] I. Cohen, M. P. Brenner, J. Eggers, and S. R. Nagel, Phys. Rev. Lett. 83, 1147 (1999).

[9] I. Cohen and S. R. Nagel, Phys. Fluids 13, 3533 (2001).

[10] A. U. Chen, P. K. Notz, and O. A. Basaran, Phys. Rev. Lett. 88, 174501 (2002).

[11] D. Leppinen and J.R. Lister, Phys. Fluids 15, 568 (2003).

[12] A. Sierou and J.R. Lister, J. Fluid Mech. 497, 381 (2003).

[13] N. C. Keim, P. Møller, W. W. Zhang, and S. R. Nagel, Bull. Am. Phys. Soc. 50, BD.00008 (2005).

[14] R. Bergmann, D. van der Meer, M. Stijnman, M. Sandtke, A. Prosperetti, and D. Lohse, Phys. Rev. Lett. 96, 154505 (2006).

[15] K. M. Sutherland, D. T. Pearson, and L. S. Gordon, Clin. Phys. Physiol. Meas. 9, 97 (1988).

[16] J. Rubio, M. L. Souza, and R. W. Smith, Miner. Eng. 15, 139 (2002).

[17] K. Theander and R. J. Pugh, Colloids Surf. A 240, 111 (2004).

[18] S. L. Liebling and M.W. Choptuik, Phys. Rev. Lett. 77, 1424 (1996).

[19] T. Plewa, A. C. Calder, and D. Q. Lamb, Astrophys. J. 612, L37 (2004).

[20] P. Doshi, I. Cohen, W. W. Zhang, M. Siegel, P. Howell, O. A. Basaran, and S. R. Nagel, Science 302, 1185 (2003).

[21] M. S. Longuet-Higgins, B. R. Kerman, and K. Lunde, J. Fluid Mech. 230, 365 (1991).

[22] H. N. Oğuz and A. Prosperetti, J. Fluid Mech. 257, 111 (1993).

[23] J. C. Burton, R. Waldrep, and P. Taborek, Phys. Rev. Lett. 94, 184502 (2005).

[24] J. M. Gordillo, A. Sevilla, J. Rodríguez-Rodríguez, and C. Martínez-Bazán, Phys. Rev. Lett. 95, 194501 (2005).

[25] D. Leppinen, J. R. Lister, and J. Eggers, Bull. Am. Phys. Soc. 50, BD.00006 (2005).

[26] S. T. Thoroddsen, T. G. Etoh, and K. Takehara, Bull. Am. Phys. Soc. 50, BD.00002 (2005).

[27] N. C. Keim, W. W. Zhang, and S. R. Nagel (unpublished).

[28] A comparison of the Bernoulli pressure, which scales as $\rho h_{\min }^{2} / \tau^{2}$ near the breakup, and the Laplace pressure, which scales as $\sigma / h_{\min }$, shows that surface tension effects are relevant in the final breakup dynamics if and only if $h_{\text {min }}$ decreases as $\tau^{2 / 3}$ or slower. 